\title{
Buildings Energy Prediction Using Artificial Neural Networks
}

\author{
Mahmoud Abdelkader Bashery Abbass ${ }^{1}$, Hatem Sadek ${ }^{2}$, Mohamed Hamdy ${ }^{3}$ \\ ${ }^{1}$ Helwan University, Department of Mechanical Power Engineering, Cairo, Egypt \\ ${ }^{2}$ Helwan University, Department of Mechanical Power Engineering, Cairo, Egypt \\ ${ }^{3}$ Department of Civil and Environmental Engineering, Norwegian University of Science and Technology, Trondheim, \\ Norway \\ mahmoud.gohar1992@m-eng.helwan.edu.eg
}

\begin{abstract}
This paper aims to prove that the artificial neural network (ANN) is a powerful tool in prediction of buildings energy consumption, this target is achieved by comparing the accuracy of ANN prediction with the output of simple linear regression algorithm and previous work. First of all, the flowchart depends on four main steps: 1) Data selection, 2) Data preparation, 3) Model training and tuning, and 4) Evaluate results. The Commercial Buildings Energy Consumption Survey (CBECS) is selected as a data set to apply ANN on it by choosing the most effective features that have the main influence on the energy consumption. Data preparation process is done by replacing missing values and outliers' values with median value of each feature. The model's hyper-parameters are tuned by manual method depending on the author expeience of ANN algorithm and the evaluation step done by using mean absolute error (MAE), mean square error (MSE), root mean square error (RMSE) and r-squared value as a metric for performance. The results showed that the proposed ANN algorithm achives high performance comparing to simple linear regression algorithm and previous work on the same data.
\end{abstract}

\section{Introduction}

ANNs are the modeling of the human brain with the simplest definition and building blocks are neurons. Each ANN consists of multilayers of neurons. A standard ANN architecture as shown in figure 1 consists of input, output, and hidden layers. The input layer takes all the input values while the output layer generates the final result [1]. The disappearance of a few pieces of information in one place does not restrict the network from functioning. After ANN training, the data may produce output even with incomplete information. ANNs have successfully used for modelling non-linear problems and complex systems [2]. When ANN gives a probing solution, it does not give a clue as to why and how. This reduces trust in the network. In addition to, there is no specific rule for determining the best structure of ANN. The appropriate network structure is achieved through experience and tune different structures using trial and 
error to achieve best performance [3-7].

ANNs is a powerful tool for modelling building energy modelling and reliable prediction. However, they require an accurate choice of network structure and precise tuning of its several hyper-parameters for training. The performance of the models is not guaranteed as ANN suffer from a local minimum problem where the solution not the best one. In addition, ANN should be fed with adequate number of samples in order to achieve acceptable accuracy. Otherwise it might be outperformed with simple MLR models. So ANN is much appropriate for engineers having a strong knowledge of deep learning and statistical modelling [3-5, 8]. The target of this paper, prove ability of using ANN algorithms in buildings energy field instead of simple linear regression algorithm and support this target by comparing results with two previous work on the same data to explain the required steps of getting high performance in prediction for energy consumption.

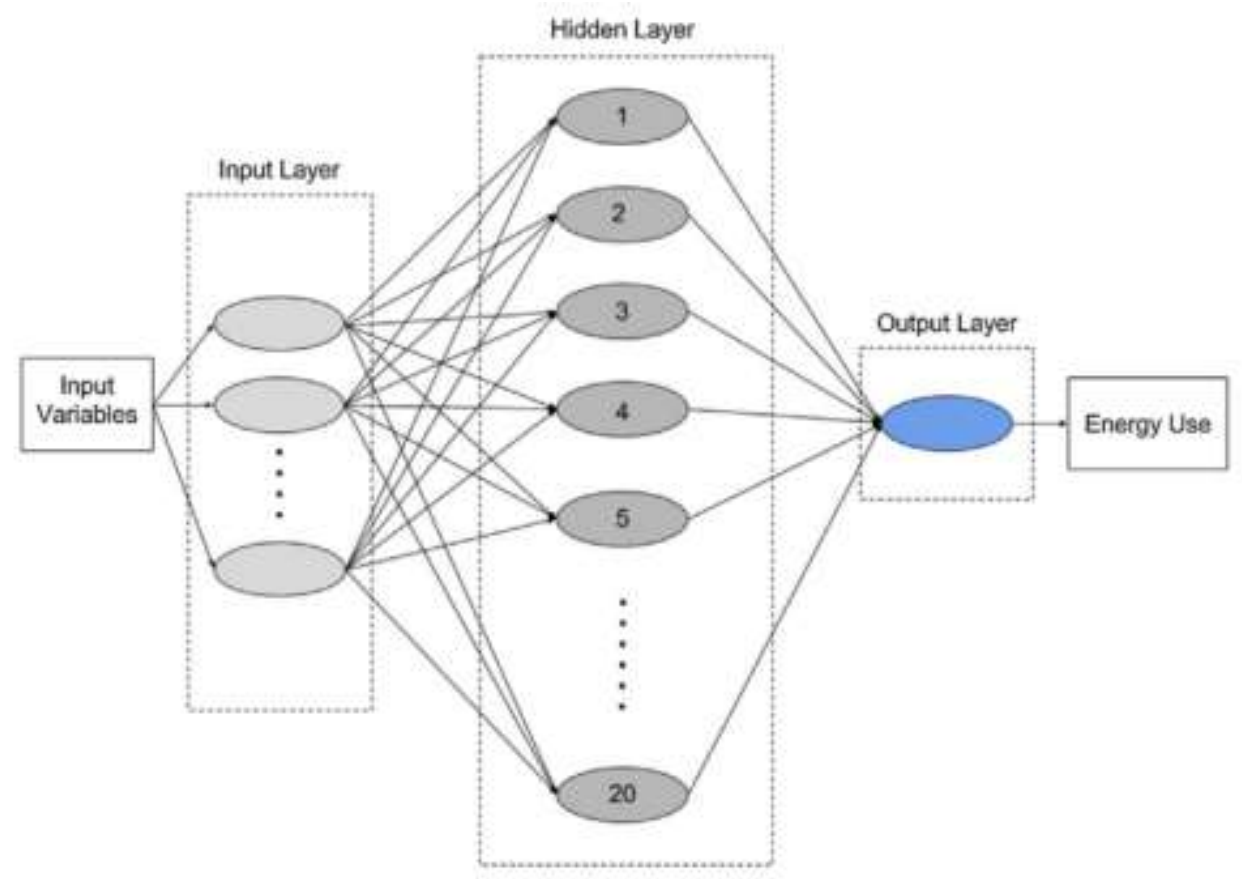

Figure 1: Artificial neural network (ANN) structure [1].

The first step to form a hidden layer is to construct hidden features based on linear combinations of the input data with next equation 1 :

$$
\mathrm{f}(\mathrm{x})=(\mathrm{W} * \mathrm{x})+\mathrm{b}
$$

where $\mathrm{W}$ represents the weight and $\mathrm{b}$ represents the bias for each neuron. Each neuron would then require a nonlinear activation function and the identity function has been used for regression purposes. The outputs of hidden layers are then used as the inputs to the output function which generates the final regression result (prediction value) [1].

In this paper, to evaluate the deviation between the predicted and actual energy use, four terms are used: 1) Mean absolute error (MAE), 2) Mean square error (MSE), 3) 
Root mean square error (RMSE), and 4) R-squared value. Equations are explained from 2 to 5 , where $n$ is the number of points in the CBECS data.

$$
\begin{aligned}
& \text { MAE }=\frac{1}{n} \sum_{i=0}^{n} \mid \text { true value }- \text { predicted value } \mid \\
& \text { MSE }=\frac{1}{n} \sum_{i=0}^{n}(\text { true value }- \text { predicted value })^{2} \\
& \mathrm{RMSE}=\sqrt{\frac{\sum_{i=0}^{n}(\text { true value }- \text { predicted value })^{2}}{n}} \\
& \mathrm{R}^{2}=1-\frac{\sum_{i=0}^{n}(\text { true value }- \text { predicted value })^{2}}{\sum_{i=0}^{n}(\text { true value-average value })^{2}}
\end{aligned}
$$

\section{Methodology}

This section represents the steps that needed to make energy prediction in building field by applying the flowchart (Figure 2) on the Commercial Buildings Energy Consumption Survey (CBECS).

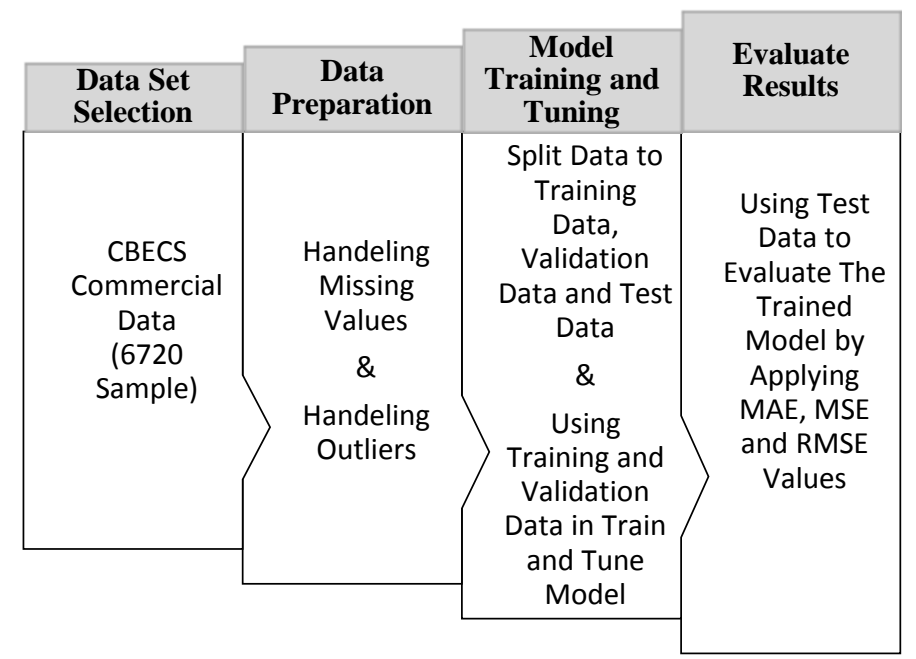

Figure 2: Artificial neural network flowchart to make building energy prediction [9, 10].

\subsection{Data Set Selection Step}

CBECS is an open source data set contain information on the stock of U.S. commercial buildings with size of 6720 building. CBECS includes building types such as schools, hospitals, correctional institutions, and buildings used for religious worship, in addition to traditional commercial buildings such as stores, restaurants, warehouses, and office buildings. In the Commercial Buildings Energy Consumption Survey (CBECS), buildings are classified according to principal activity, which is the primary business, commerce, or function carried on within each building $[11,12]$.

\subsection{Data Set Preparation Steps}

After choosing suitable data set to test the artificial neural network algorithm, data preprocessing step comes here. The numerical features are selected in addition to 
the main categorical features that effect on energy consumption in direct way or indirect way. The target during analysis is checking missing values, outlier values and understanding the nature of each feature distribution. The selected features are shown in (Table 1 and Table 2) which can be classified as: 1) Categorical features, and 2) Numerical features.

The categorical features are converted directly to one hot encoder after get rid of outliers and missing values in it. But in case of numerical features the preprocessing step need for calculation of mean and median for each feature (Table 2) to show the difference between mean and median that represent the effect of outliers on data distribution, in addition to calculate the percentage of missing values in each feature to identify how to deal with it. The change of the difference value represents the effect of outliers; the more outliers effect increases the more difference between mean and median increase. The visualization of missing values of each feature is very important to take a decision of which feature is suitable to taken in training neural network, because features with high percentage of missing values can't be taken.

Table 1: categories, percentage of each category of total data and percentage of missing values for each categorical feature

\begin{tabular}{|c|c|c|c|c|c|}
\hline & $\begin{array}{c}\text { Variable } \\
\text { name }\end{array}$ & Label & Categories Numbers & $\begin{array}{l}\text { Percentage of Each Category of Total } \\
\text { Data } \\
\text { (sorted from lowest to highest value) }\end{array}$ & $\begin{array}{l}\text { Percentage } \\
\text { of Missing } \\
\text { Values }\end{array}$ \\
\hline 1 & CENDIV & $\begin{array}{l}\text { Census } \\
\text { division } \\
\text { (Categorical } \\
\text { Feature) }\end{array}$ & $\begin{array}{l}\text { '1' = 'New England' } \\
\text { '2' = 'Middle Atlantic' } \\
\text { '3' = 'East North Central' } \\
\text { '4' = 'West North Central' } \\
\text { '5' = 'South Atlantic' } \\
\text { '6' = 'East South Central' } \\
\text { '7' = 'West South Central' } \\
\text { '8' = 'Mountain' } \\
\text { '9' = 'Pacific' }\end{array}$ & $\begin{array}{l}\text { New England = 4.75 } \\
\text { East South Central = 5.83 } \\
\text { Mountain }=6.70 \\
\text { West North Central = 8.32 } \\
\text { Middle Atlantic = } 11.71 \\
\text { West South Central = 12.68 } \\
\text { East North Central = 13.39 } \\
\text { Pacific = 16.56 } \\
\text { South Atlantic = 20.06 }\end{array}$ & 0.00 \\
\hline r & PBA & $\begin{array}{c}\text { Principal } \\
\text { building } \\
\text { activity } \\
\text { (Categorical } \\
\text { Feature) }\end{array}$ & $\begin{array}{l}\text { '01' = 'Vacant' } \\
\text { '02' = 'Office' } \\
\text { '04' = 'Laboratory' } \\
\text { '05' = 'Non-refrigerated warehouse' } \\
\text { '06' = 'Food sales' } \\
\text { '07' = 'Public order and safety' } \\
\text { '08' = 'Outpatient health care' } \\
\text { '11' = 'Refrigerated warehouse' } \\
\text { '12' = 'Religious worship' } \\
\text { '13' = 'Public assembly' } \\
\text { '14' = 'Education' } \\
\text { '15' = 'Food service' } \\
\text { '16' = 'Inpatient health care' } \\
\text { '17' = 'Nursing' } \\
\text { '18' = 'Lodging' } \\
\text { '23' = 'Strip shopping mall' } \\
\text { '24' = 'Enclosed mall' } \\
\text { '25' = 'Retail other than mall' } \\
\text { '26' = 'Service' } \\
\text { '27' = 'Other' }\end{array}$ & $\begin{array}{l}\text { Refrigerated warehouse }=0.31 \\
\text { Enclosed mall }=0.51 \\
\text { Laboratory }=0.61 \\
\text { Other }=1.29 \\
\text { Nursing }=1.40 \\
\text { Public order and safety }=1.50 \\
\text { Food sales }=1.92 \\
\text { Outpatient health care }=2.93 \\
\text { Vacant }=3.68 \\
\text { Lodging }=4.39 \\
\text { Strip shopping mall = 4.40 } \\
\text { Religious worship = 5.24 } \\
\text { Food service }=5.37 \\
\text { Retail other than mall }=5.49 \\
\text { Inpatient health care }=6.09 \\
\text { Public assembly }=6.16 \\
\text { Service }=6.31 \\
\text { Non-refrigerated warehouse }=10.98 \\
\text { Education }=11.24 \\
\text { Office }=20.18\end{array}$ & 0.00 \\
\hline$r$ & WLCNS & $\begin{array}{c}\text { Wall } \\
\text { construction }\end{array}$ & $\begin{array}{l}\text { '1' = 'Brick, stone, or stucco' } \\
\text { '2' = 'Pre-cast concrete panels' }\end{array}$ & $\begin{array}{l}\text { Decorative or construction glass }=0.31 \\
\text { No one major type }=0.61\end{array}$ & 0.00 \\
\hline
\end{tabular}




\begin{tabular}{|c|c|c|c|c|c|}
\hline & & $\begin{array}{c}\text { material } \\
\text { (Categorical } \\
\text { Feature) }\end{array}$ & $\begin{array}{l}\text { '3' = 'Concrete block or poured concrete } \\
\text { (above grade)' } \\
\text { '4' = 'Aluminum, asbestos, plastic, or } \\
\text { wood materials (siding, shingles, tiles, or } \\
\text { shakes)' } \\
\text { '5' = 'Sheet metal panels' } \\
\text { '6' = 'Window or vision glass (glass that } \\
\text { can be seen through)' } \\
\text { '7' = 'Decorative or construction glass' } \\
\text { '8' = 'No one major type' } \\
\text { '9' = 'Other' }\end{array}$ & $\begin{array}{l}\text { Other }=0.62 \\
\text { Window or vision glass (glass that can be } \\
\text { seen through) }=1.53 \\
\text { Pre-cast concrete panels }=8.54 \\
\text { Aluminum, asbestos, plastic, or wood } \\
\text { materials (siding, shingles, tiles, or } \\
\text { shakes) }=9.48 \\
\text { Sheet metal panels }=9.93 \\
\text { Concrete block or poured concrete (above } \\
\text { grade) }=21.80 \\
\text { Brick, stone, or stucco }=47.17\end{array}$ & \\
\hline$\varepsilon$ & BLDSHP & $\begin{array}{l}\text { Building } \\
\text { shape } \\
\text { (Categorical } \\
\text { Feature) }\end{array}$ & $\begin{array}{l}\text { '01' = 'Square' } \\
\text { '02' = 'Wide rectangle' } \\
\text { '03' = 'Narrow rectangle' } \\
\text { '04' = 'Rectangle or square with an } \\
\text { interior courtyard' } \\
\text { '05' = "'H" shaped' } \\
\text { '06' = '"U" shaped' } \\
\text { '07' = '"E" shaped' } \\
\text { '08' = '"T" shaped' } \\
\text { '09' = '"L" shaped' } \\
\text { '10' = '"+" or cross shaped' } \\
' 11 \text { = 'Other shape' }\end{array}$ & $\begin{array}{l}\text { "E" shaped }=0.97 \\
\text { "H" shaped }=1.72 \\
\text { "+" or cross shaped }=1.74 \\
\text { "T" shaped }=2.03 \\
\text { "U" shaped }=2.24 \\
\text { Rectangle or square with an interior } \\
\text { courtyard }=3.72 \\
\text { "L" shaped }=6.06 \\
\text { Other shape }=6.13 \\
\text { Narrow rectangle }=7.27 \\
\text { Square }=7.91 \\
\text { Wide rectangle }=60.22\end{array}$ & 9.14 \\
\hline 0 & MONCON & $\begin{array}{c}\text { Month ready } \\
\text { for occupancy } \\
\text { (Categorical } \\
\text { Feature) }\end{array}$ & $\begin{array}{l}\text { '01' = 'January' } \\
\text { '02' = 'February' } \\
\text { '03' = 'March' } \\
\text { '04' = 'April' } \\
\text { '05' = 'May' } \\
\text { '06' = 'June' } \\
\text { '07' = 'July' } \\
\text { '08' = 'August' } \\
\text { '09' = 'September' } \\
\text { '10' = 'October' } \\
\text { '11' = 'November' } \\
\text { '12' = 'December' } \\
\text { '00' = 'Undetermined' }\end{array}$ & $\begin{array}{l}\text { February }=1.72 \\
\text { January }=3.45 \\
\text { June }=3.45 \\
\text { September }=5.17 \\
\text { November }=5.17 \\
\text { July }=6.90 \\
\text { March }=6.90 \\
\text { August }=8.62 \\
\text { April }=10.34 \\
\text { October }=10.34 \\
\text { May }=10.34 \\
\text { Undetermined }=12.07 \\
\text { December }=15.52\end{array}$ & 99.14 \\
\hline 7 & ACT1 & $\begin{array}{c}\text { First activity } \\
\text { in building } \\
\text { (Categorical } \\
\text { Feature) }\end{array}$ & \multirow{2}{*}{$\begin{array}{l}\text { '11' = 'Office/Professional' } \\
\text { '12' = 'Data center/Computer "server } \\
\text { farm"' } \\
\text { '13' = 'Warehouse/Storage' } \\
\text { '14' = 'Food sales or service' } \\
\text { '15' = 'Enclosed mall' } \\
\text { '16' = 'Retail (other than mall)' } \\
\text { '17' = 'Education' } \\
\text { '18' = 'Religious worship' } \\
\text { '19' = 'Public assembly' } \\
\text { '20' = 'Health care' } \\
\text { '21' = 'Service' } \\
\text { '22' = 'Lodging' } \\
\text { '23' = 'Public order and safety' } \\
\text { '24' = 'Residential' } \\
\text { '25' = 'Industrial' } \\
\text { '26' = 'Agricultural' } \\
\text { '27' = 'Vacant' } \\
\text { '28' = 'Other' }\end{array}$} & $\begin{array}{l}\text { Industrial }=0.27 \\
\text { Other }=0.27 \\
\text { Lodging }=0.27 \\
\text { Residential }=0.27 \\
\text { Religious worship =0.53 } \\
\text { Public assembly =0.53 } \\
\text { Service }=0.80 \\
\text { Health care }=1.06 \\
\text { Retail }(\text { other than mall })=4.24 \\
\text { Education }=6.63 \\
\text { Food sales or service = } 8.49 \\
\text { Warehouse/Storage = } 11.41 \\
\text { Office/Professional = 65.25 }\end{array}$ & 94.39 \\
\hline v & ACT2 & $\begin{array}{l}\text { Second } \\
\text { activity in } \\
\text { building } \\
\text { (Categorical } \\
\text { Feature) }\end{array}$ & & $\begin{array}{l}\text { Public order and safety }=1.86 \\
\text { Industrial }=2.65 \\
\text { Vacant }=2.92 \\
\text { Lodging }=2.92 \\
\text { Residential }=4.77 \\
\text { Religious worship }=5.04 \\
\text { Service }=6.10 \\
\text { Health care }=6.10 \\
\text { Other }=6.90 \\
\text { Food sales or service = } 7.69 \\
\text { Education }=7.69 \\
\text { Public assembly }=10.08 \\
\end{array}$ & 94.39 \\
\hline
\end{tabular}




\begin{tabular}{|c|c|c|c|c|c|}
\hline & & & & $\begin{array}{l}\text { Retail }(\text { other than mall })=11.94 \\
\text { Warehouse/Storage }=23.34\end{array}$ & \\
\hline$\wedge$ & ACT3 & $\begin{array}{l}\text { Third activity } \\
\text { in building } \\
\text { (Categorical } \\
\text { Feature) }\end{array}$ & & $\begin{array}{l}\text { Food sales or service }=1.16 \\
\text { Lodging }=2.33 \\
\text { Education }=2.33 \\
\text { Religious worship }=3.49 \\
\text { Public order and safety = } 3.49 \\
\text { Health care }=6.40 \\
\text { Vacant }=6.40 \\
\text { Residential }=7.56 \\
\text { Retail }(\text { other than mall })=8.72 \\
\text { Industrial = } 12.79 \\
\text { Other }=13.37 \\
\text { Public assembly = } 14.53 \\
\text { Service = } 17.44\end{array}$ & 97.44 \\
\hline$q$ & PBAPLUS & $\begin{array}{c}\text { More specific } \\
\text { building } \\
\text { activity } \\
\text { (Categorical } \\
\text { Feature) }\end{array}$ & $\begin{array}{l}\text { '01' = 'Vacant' } \\
\text { '02' = 'Administrative/professional } \\
\text { office' } \\
\text { '03' = 'Bank/other financial' } \\
\text { '04' = 'Government office' } \\
\text { '05' = 'Medical office (non-diagnostic)' } \\
\text { '06' = 'Mixed-use office' } \\
\text { '07' = 'Other office' } \\
\text { '08' = 'Laboratory' } \\
\text { '09' = 'Distribution/shipping center' } \\
\text { '10' = 'Non-refrigerated warehouse' } \\
\text { '11' = 'Self-storage' } \\
\text { '12' = 'Convenience store' } \\
\text { '13' = 'Convenience store with gas } \\
\text { station' } \\
\text { '14' = 'Grocery store/food market' } \\
\text { '15' = 'Other food sales' } \\
\text { '16' = 'Fire station/police station' } \\
\text { '17' = 'Other public order and safety' } \\
\text { '18' = 'Medical office (diagnostic)' } \\
\text { '19' = 'Clinic/other outpatient health' } \\
\text { '20' = 'Refrigerated warehouse' } \\
\text { '21' = 'Religious worship' } \\
\text { '22' = 'Entertainment/culture' } \\
\text { '23' = 'Library' } \\
\text { '24' = 'Recreation' } \\
\text { '25' = 'Social/meeting' } \\
\text { '26' = 'Other public assembly' } \\
\text { '27' = 'College/university' } \\
\text { '28' = 'Elementary/middle school' } \\
\text { '29' = 'High school' } \\
\text { '30' = 'Preschool/daycare' } \\
\text { '31' = 'Other classroom education' } \\
\text { '32' = 'Fast food' } \\
\text { '33' = 'Restaurant/cafeteria' } \\
\text { '34' = 'Other food service' } \\
\text { '35' = 'Hospital/inpatient health' } \\
\text { '36' = 'Nursing home/assisted living' } \\
\text { '37' = 'Dormitory/fraternity/sorority' } \\
\text { '38' = 'Hotel' } \\
\text { '39' = 'Motel or inn' } \\
\text { '40' = 'Other lodging' } \\
41 \text { ' = 'Vehicle dealership/showroom' }\end{array}$ & $\begin{array}{l}\text { Other food sales }=0.03 \\
\text { Refrigerated warehouse }=0.31 \\
\text { Other public order and safety }=0.33 \\
\text { Courthouse/probation office }=0.39 \\
\text { Post office/postal center }=0.39 \\
\text { Other lodging }=0.40 \\
\text { Other food service }=0.40 \\
\text { Convenience store with gas station }=0.48 \\
\text { Vehicle dealership/showroom }=0.51 \\
\text { Enclosed mall }=0.51 \\
\text { Library }=0.55 \\
\text { Other retail }=0.61 \\
\text { Laboratory }=0.61 \\
\text { Medical office }(\text { non-diagnostic })=0.62 \\
\text { Convenience store }=0.70 \\
\text { Grocery store/food market }=0.71 \\
\text { Dormitory/fraternity/sorority }=0.71 \\
\text { Preschool/daycare }=0.74 \\
\text { Other office }=0.77 \\
\text { Fire station/police station }=0.79 \\
\text { Repair shop }=0.79 \\
\text { Bar/pub/lounge }=0.89 \\
\text { Motel or inn }=0.91 \\
\text { Medical office }(\text { diagnostic })=0.92 \\
\text { Other classroom education }=0.92 \\
\text { Other public assembly }=0.94 \\
\text { Bank/other financial }=1.18 \\
\text { Self-storage }=1.21 \\
\text { Other service }=1.24 \\
\text { Other }=1.29 \\
\text { Entertainment/culture }=1.32 \\
\text { Fast food }=1.40 \\
\text { Nursing home/assisted living }=1.40 \\
\text { Social/meeting }=1.46 \\
\text { College/university }=1.55 \\
\text { Vehicle storage/maintenance }=1.68 \\
\text { Recreation }=1.89 \\
\text { Clinic/other outpatient health }=2.01 \\
\text { High school }=2.11 \\
\text { Vehicle service/repair shop }=2.22 \\
\text { Hotel }=2.37 \\
\text { Restaurant/cafeteria }=2.68 \\
\text { Government office }=3.05 \\
\end{array}$ & 0.00 \\
\hline
\end{tabular}




\begin{tabular}{|c|c|c|c|c|c|}
\hline & & & $\begin{array}{l}\text { '42' = 'Retail store' } \\
\text { '43' = 'Other retail' } \\
\text { '44' = 'Post office/postal center' } \\
\text { '45' = 'Repair shop' } \\
\text { '46' = 'Vehicle service/repair shop' } \\
\text { '47' = 'Vehicle storage/maintenance' } \\
\text { '48' = 'Other service' } \\
\text { '49' = 'Other' } \\
\text { '50' = 'Strip shopping mall' } \\
\text { '51' = 'Enclosed mall' } \\
\text { '52' = 'Courthouse/probation office' } \\
53 \text { ' = 'Bar/pub/lounge' }\end{array}$ & $\begin{array}{l}\text { Mixed-use office }=3.15 \\
\text { Vacant }=3.68 \\
\text { Retail store }=4.38 \\
\text { Strip shopping mall }=4.40 \\
\text { Distribution/shipping center }=4.57 \\
\text { Non-refrigerated warehouse }=.21 \\
\text { Religious worship }=5.24 \\
\text { Elementary/middle school }=5.91 \\
\text { Hospital/inpatient health }=6.09 \\
\text { Administrative/professional office = } \\
11.40\end{array}$ & \\
\hline 1. & FKTYPE & $\begin{array}{l}\text { Specify fuel } \\
\text { oil, diesel, or } \\
\text { kerosene } \\
\text { (Categorical } \\
\text { Feature) }\end{array}$ & $\begin{array}{l}\text { '1' = 'Fuel oil' } \\
\text { '2' = 'Diesel' } \\
\text { '3' = 'Kerosene' } \\
\text { '4' = 'Fuel oil and diesel' } \\
\text { '5' = 'Fuel oil and kerosene' } \\
\text { '6' = 'Diesel and kerosene' } \\
\text { '7' = 'Fuel oil, diesel, and kerosene' } \\
\text { '9' ='Don't know' }\end{array}$ & $\begin{array}{l}\text { Diesel and kerosene }=0.13 \\
\text { Fuel oil and kerosene }=0.20 \\
\text { Fuel oil, diesel, and kerosene }=0.86 \\
\text { Kerosene }=1.52 \\
\text { Fuel oil and diesel }=4.02 \\
\text { Don't know }=18.34 \\
\text { Fuel oil }=23.35 \\
\text { Diesel }=51.58\end{array}$ & 77.44 \\
\hline
\end{tabular}

Table 2: minimum, mean, median, maximum and percentage of missing values for each numerical feature

\begin{tabular}{|c|c|c|c|c|c|c|c|}
\hline & $\begin{array}{l}\text { Variable } \\
\text { name }\end{array}$ & Label & Minimum Value & Mean Values & Median Values & $\begin{array}{l}\text { Maximum } \\
\text { Value }\end{array}$ & $\begin{array}{c}\text { Percentage } \\
\text { of Missing } \\
\text { Values }\end{array}$ \\
\hline 1 & SQFT & Square footage area & 1001 & 124473.50 & 20750.00 & 1500000 & 0.00 \\
\hline 2 & NFLOOR & Number of floors & 1 & 3.00 & 2.00 & 30 & 0.00 \\
\hline 3 & BASEMNT & $\begin{array}{c}\text { Number of } \\
\text { underground floors }\end{array}$ & 0 & 0.34 & 0.0 & 7 & 49.63 \\
\hline 4 & FLCEILHT & $\begin{array}{l}\text { Floor to ceiling } \\
\text { height (foot) }\end{array}$ & 6 & 12.87 & 10.00 & 60 & 0.00 \\
\hline 5 & NELVTR & Number of elevators & 1 & 12.45 & 3.00 & 60 & 67.74 \\
\hline 6 & NESLTR & Number of escalators & 1 & 6.39 & 4.00 & 24 & 97.28 \\
\hline 7 & YRCON & Year of construction & 1932 & 1977.00 & 1981.00 & 2012 & 0.00 \\
\hline 8 & ACT1PCT & $\begin{array}{c}\text { Percent used for first } \\
\text { activity }\end{array}$ & 0 & 2.13 & 0.00 & 100 & 94.39 \\
\hline 9 & АCT2PCT & $\begin{array}{l}\text { Percent used for } \\
\text { second activity }\end{array}$ & 0 & 41.29 & 45.00 & 100 & 94.39 \\
\hline 10 & АСТЗРСТ & $\begin{array}{c}\text { Percent used for third } \\
\text { activity }\end{array}$ & 0 & 33.53 & 33.00 & 100 & 97.44 \\
\hline 11 & RWSEAT & $\begin{array}{l}\text { Religious worship } \\
\text { seating capacity }\end{array}$ & 0 & 22.01 & 0.00 & 1800 & 94.76 \\
\hline 12 & PBSEAT & $\begin{array}{l}\text { Assembly seating } \\
\text { capacity }\end{array}$ & 0 & 88.26 & 0.00 & 18000 & 93.84 \\
\hline 13 & EDSEAT & $\begin{array}{c}\text { Number of classroom } \\
\text { seats }\end{array}$ & 0 & 70.00 & 0.00 & 8000 & 88.76 \\
\hline
\end{tabular}


Hatem Sadek/ Engineering Research Journal 171 (septamper 2021) M20- M32

\begin{tabular}{|c|c|c|c|c|c|c|c|}
\hline 14 & FDSEAT & $\begin{array}{l}\text { Food service seating } \\
\text { capacity }\end{array}$ & 0 & 5.81 & 0.00 & 700 & 94.63 \\
\hline 15 & HCBED & $\begin{array}{l}\text { Licensed bed } \\
\text { capacity }\end{array}$ & 0 & 13.70 & 0.00 & 300 & 93.91 \\
\hline 16 & LODGRM & $\begin{array}{l}\text { Number of guest } \\
\text { rooms }\end{array}$ & 0 & 8.38 & 0.00 & 1080 & 95.61 \\
\hline 17 & NOCC & Number of businesses & 0 & 3.90 & 1.00 & 240 & 0.00 \\
\hline 18 & MONUSE & Months in use & 0 & 11.32 & 12.00 & 12 & 0.00 \\
\hline 19 & OCCUPYP & Percent occupancy & 0 & 25.00 & 0.00 & 100 & 71.98 \\
\hline 20 & LODOCCP & $\begin{array}{l}\text { Lodging room } \\
\text { percent occupancy }\end{array}$ & 0 & 2.27 & 0.00 & 100 & 96.56 \\
\hline 21 & WKHRS & $\begin{array}{c}\text { Total hours open per } \\
\text { week }\end{array}$ & 0 & 78.02 & 60.00 & 168 & 0.00 \\
\hline 22 & NWKER & $\begin{array}{l}\text { Number of } \\
\text { employees }\end{array}$ & 0 & 178.78 & 15.00 & 6500 & 0.00 \\
\hline 23 & HEATP & Percent heated & 0 & 81.49 & 100.00 & 100 & 7.95 \\
\hline 24 & COOLP & Percent cooled & 0 & 71.68 & 95.00 & 100 & 10.18 \\
\hline 25 & BOOSTWT & Booster water heaters & 0 & 0.11 & 0.00 & 60 & 93.63 \\
\hline 26 & XRAYN & $\begin{array}{c}\text { Number of X-ray } \\
\text { machines }\end{array}$ & 0 & 0.59 & 0.00 & 24 & 89.88 \\
\hline 27 & RFGRSN & $\begin{array}{c}\text { Number of residential } \\
\text { refrigerators }\end{array}$ & 0 & 6.30 & 1.00 & 1000 & 40.43 \\
\hline 28 & RFGCOMPN & $\begin{array}{l}\text { Number of compact } \\
\text { refrigerators }\end{array}$ & 0 & 9.00 & 0.00 & 1200 & 62.16 \\
\hline 29 & RFGWIN & $\begin{array}{l}\text { Number of walk-in } \\
\text { units }\end{array}$ & 0 & 1.24 & 0.00 & 350 & 69.32 \\
\hline 30 & RFGOPN & $\begin{array}{l}\text { Number of open case } \\
\text { refrigeration units }\end{array}$ & 0 & 1.21 & 0.00 & 300 & 85.65 \\
\hline 31 & RFGCLN & $\begin{array}{c}\text { Number of closed } \\
\text { case refrigeration } \\
\text { units }\end{array}$ & 0 & 2.44 & 0.00 & 500 & 72.62 \\
\hline 32 & RFGVNN & $\begin{array}{c}\text { Number of } \\
\text { refrigerated vending } \\
\text { machines }\end{array}$ & 0 & 2.24 & 0.00 & 300 & 58.79 \\
\hline 33 & RFGICN & $\begin{array}{c}\text { Number of ice } \\
\text { makers }\end{array}$ & 0 & 2.70 & 0.00 & 500 & 66.28 \\
\hline 34 & RFGSTP & Percent cold storage & 0 & 0.50 & 0.00 & 100 & 96.24 \\
\hline 35 & PCTERMN & Number of computers & 0 & 162.92 & 9.00 & 4195 & 3.56 \\
\hline 36 & LAPTPN & Number of laptops & 0 & 53.00 & 2.00 & 1420 & 3.56 \\
\hline 37 & PRNTRN & Number of printers & 0 & 49.58 & 4.00 & 5000 & 13.13 \\
\hline 38 & SERVERN & Number of servers & 0 & 9.49 & 1.00 & 600 & 3.56 \\
\hline
\end{tabular}




\begin{tabular}{|c|c|c|c|c|c|c|c|c|}
\hline 39 & TVVIDEON & $\begin{array}{l}\text { Number of TV or } \\
\text { video displays }\end{array}$ & 0 & & 25.51 & 2.00 & 240 & 32.13 \\
\hline 40 & RGSTRN & $\begin{array}{l}\text { Number of cash } \\
\text { registers }\end{array}$ & 0 & & 3.95 & 0.00 & 500 & 55.16 \\
\hline 41 & COPIERN & $\begin{array}{c}\text { Number of } \\
\text { photocopiers }\end{array}$ & 0 & & 10.51 & 1.00 & 1500 & 40.18 \\
\hline 42 & LTOHRP & Percent lit when open & 0 & & 78.50 & 90.00 & 100 & 4.40 \\
\hline 43 & LTNHRP & Percent lit off hours & 0 & & 15.17 & 5.00 & 100 & 4.97 \\
\hline 44 & DAYLTP & Percent daylight & 0 & & 17.35 & 5.00 & 100 & 3.68 \\
\hline 45 & MFBTU & $\begin{array}{c}\text { Annual major fuel } \\
\text { consumption } \\
\text { (thousands Btu) }\end{array}$ & \multirow{8}{*}{$\begin{array}{c}\text { Chosen as } \\
\text { Output } \\
\text { Features } \\
\text { for ANN } \\
\text { Model }\end{array}$} & 3.0 & 17465901.90 & 39292406.0 & 1481866360.0 & 2.47 \\
\hline 46 & MFEXP & $\begin{array}{l}\text { Annual major fuel } \\
\text { expenditures } \\
(\$)\end{array}$ & & 4.0 & 336921.15 & 618898.00 & 36471871.0 & 2.47 \\
\hline 47 & ELBTU & $\begin{array}{c}\text { Annual electricity } \\
\text { consumption } \\
\text { (thousands Btu) }\end{array}$ & & 0.0 & 9283680.98 & 26182500.5 & 1360596256.0 & 2.47 \\
\hline 48 & ELEXP & $\begin{array}{c}\text { Annual electricity } \\
\text { expenditures } \\
\text { (\$) }\end{array}$ & & 0.0 & 250045.54 & 669039.5 & 35740206.0 & 2.47 \\
\hline 49 & NGBTU & $\begin{array}{l}\text { Annual natural gas } \\
\text { consumption } \\
\text { (thousands Btu) }\end{array}$ & & 103.0 & 8627255.97 & 742971.5 & 1225754143.0 & 35.06 \\
\hline 50 & NGEXP & $\begin{array}{c}\text { Annual natural gas } \\
\text { expenditures } \\
(\$)\end{array}$ & & 4.0 & 60373.00 & 5570.0 & 14501641.0 & 35.06 \\
\hline 51 & FKBTU & $\begin{array}{l}\text { Annual fuel oil } \\
\text { consumption } \\
\text { (thousands Btu) }\end{array}$ & & 0.0 & 1156019.06 & 208826.00 & 96306196.0 & 77.46 \\
\hline 52 & FKEXP & $\begin{array}{l}\text { Annual fuel oil } \\
\text { expenditures } \\
\text { (\$) }\end{array}$ & & 0.0 & 27926.54 & 5374.00 & 2041370.0 & 77.46 \\
\hline
\end{tabular}

After feature visualization, the problems of missing values and outliers can be handled by making some changes: 1) The features that have missing values more than $75 \%$ will be deleted, 2) The features that have low value of missing values is imputed by the median value of feature distribution, and 3) The outliers' values will be replaced by median values. Finally, the data is normalized with respect to maximum value of each feature, the normalization is very important to train weights of neural network [11].

\subsection{Model Training and Tuning}

After data preparation the step of training ANN weights comes here. So, the data set is separated to three packages: 1) Training data to train model weights, 2) Validation data to tune model weights, and 3) Test data or unseen data to evaluate 
the trained model. The performance of ANN is depending mainly on model's parameters which called hyper-parameters such as: 1) The learning rate, 2) The number of denes layer, 3) The number of nodes per layer, 4) The loss function, 5) The nodes' activation functions, 6) The metric function, and 7) The Optimizer. The aim of the hyper-parameters tuning step is to find the best hyper-parameters values that return best performance on test data set. To find the best hyper-parameters values by try and error method without consuming time is need experience in the field [13-16].

The trial and error method is used to choose best hyper-parameters for ANN concluded with: 1) Learning rate equal to $4.69 \mathrm{e}-05$; 2) Dense layers equal to 7 ; 3) Dense nodes per layer equal to 277 ; 4) Loss function is mean square error (MSE); 5) Activation function for nodes is the rectified linear unit (ReLU); 6) The metric function is mean absolute percentage error which known as best metric function option for prediction energy consumption [17]; and 7) The optimizer selected for training is Adam optimizer because it was found to have significant results with different values of learning rate and different problems in building energy applications $[18,19]$.

\subsection{Evaluate Results}

The selected metric to evaluate model is mean absolute error (MAE), mean square error (MSE), root mean square error (RMSE) and r-squared value. The trained ANN model results are $0.004323,0.000152,0.012312$ and 0.933945 respectively (Table r). The proposed ANN model is also evaluated by comparing to simple linear regression algorithm which give results of $0.0045,0.000232,0.015238$ and 0.635904 respectively.

Table 3: Comparison between simple linear regression, proposed $\mathrm{ANN}$ results and previous work results

\begin{tabular}{|c|c|c|c|c|c|}
\hline & Algorithm & $\begin{array}{c}\text { Simple } \\
\text { Linear } \\
\text { Regression }\end{array}$ & $\begin{array}{c}\text { Proposed } \\
\text { ANN }\end{array}$ & $\begin{array}{c}\text { Previous ANN } \\
{[11]}\end{array}$ & $\begin{array}{c}\text { Previous ANN } \\
{[20]}\end{array}$ \\
\hline & $\begin{array}{l}\text { umber of Input } \\
\text { Features }\end{array}$ & & & 8 & 20 \\
\hline & $\begin{array}{l}\text { mber of Output } \\
\text { Features }\end{array}$ & & & 1 & 1 \\
\hline & $\begin{array}{l}\text { Mean Absolute } \\
\text { Error }\end{array}$ & $0.45 \%$ & $0.4323 \%$ & $\begin{array}{c}\text { Not Used in } \\
\text { Original } \\
\text { Paper }\end{array}$ & $\begin{array}{c}\text { Not Used in } \\
\text { Original } \\
\text { Paper }\end{array}$ \\
\hline$\sum_{=}^{\infty}$ & $\begin{array}{l}\text { Mean Square } \\
\text { Error }\end{array}$ & $0.0232 \%$ & $0.0152 \%$ & $9.6 \%$ & $\begin{array}{c}\text { Not Used in } \\
\text { Original } \\
\text { Paper }\end{array}$ \\
\hline 承 & $\begin{array}{l}\text { Root Mean } \\
\text { Square Error }\end{array}$ & $1.5238 \%$ & $1.2312 \%$ & $\begin{array}{c}\text { Not Used in } \\
\text { Original } \\
\text { Paper }\end{array}$ & $\begin{array}{c}\text { Not Used in } \\
\text { Original } \\
\text { Paper }\end{array}$ \\
\hline & R-Squared & 0.635904 & 0.933945 & $\begin{array}{c}\text { Not Used in } \\
\text { Original } \\
\text { Paper }\end{array}$ & 0.82 \\
\hline
\end{tabular}


The comparison declared in (Table 3) is showing the power of proposed ANN, especially in comparison with previous work on same data. The main drawback in the previous work that reduce performance is the conversion of all numerical feature to categories which reduce accuracy of predicting specific value, in addition to they drop some important features without study the effect of each one on the output features. In this paper the data is handled as a combination between numerical and categorical features, so the model gives low error and high prediction accuracy comparing to linear regression and previous work on the same data.

\section{Conclusion}

This paper introduce description for ANN implementation in buildings energy prediction field which have high degree of complexity because of the non-linear relation between features in the field. The ANN algorithm have high degree of flexibility to deal with different cases by handling hyper-parameters of it. The proposed ANN algorithm have hyper-parameter values as following (learning rate value is $4.6895 \mathrm{e}-05$, number of dense layers is 7 , number of dense nodes per layer is 277, loss function is MSE and activation function is (ReLU). This hyper-parameters values give high performance comparing to simple linear regression algorithm for prediction of multi-output values where the trained ANN model results are 0.0045 for MAE, 0.000232 for MSE, 0.015238 for RMSE and 0.933945 for $r$-square value while the simple linear regression algorithm gives results of 0.0045 for MAE, 0.000232 for MSE, 0.015238 for RMSE and 0.635904 for r-square value. In addition, the comparison between proposed model and previous work on the same data declare the high performance of the proposed one. The conclusion declare that the proposed ANN model is a powerful prediction tool to be used in the energy and building field.

\section{References}

1. Deng, H., D. Fannon, and M.J. Eckelman, Predictive modeling for US commercial building energy use: A comparison of existing statistical and machine learning algorithms using CBECS microdata. Energy and Buildings, 2018. 163: p. 34-43.

2. Yang, I.-H., M.-S. Yeo, and K.-W. Kim, Application of artificial neural network to predict the optimal start time for heating system in building. Energy Conversion and Management, 2003. 44(17): p. 2791-2809.

3. Aydinalp, M., V. Ismet Ugursal, and A.S. Fung, Modeling of the space and domestic hotwater heating energy-consumption in the residential sector using neural networks. Applied Energy, 2004. 79(2): p. 159-178.

4. Edwards, R.E., J. New, and L.E. Parker, Predicting future hourly residential electrical consumption: A machine learning case study. Energy and Buildings, 2012. 49: p. 591-603.

5. Kialashaki, A. and J.R. Reisel, Modeling of the energy demand of the residential sector in the United States using regression models and artificial neural networks. Applied Energy, 2013. 108: p. 271-280.

6. Soteris A. Kalogirou, M.B., Artificial neural networks for the prediction of the energy consumption of a passive solar building. Energy, 1999. 
7. T. Olofsson, S.A., Long-term energy demand predictions based on short-term measured data. Energy and Buildings, 2001.

8. Hong, S.-M., et al., Improved benchmarking comparability for energy consumption in schools. Building Research \& Information, 2013. 42(1): p. 47-61.

9. Fayaz, M. and D. Kim, A Prediction Methodology of Energy Consumption Based on Deep Extreme Learning Machine and Comparative Analysis in Residential Buildings. Electronics, 2018. 7(10).

10. Liu, Z., et al., Accuracy analyses and model comparison of machine learning adopted in building energy consumption prediction. Energy Exploration \& Exploitation, 2019. 37(4): p. 1426-1451.

11. Yalcintas, M. and U. Aytun Ozturk, An energy benchmarking model based on artificial neural network method utilizing US Commercial Buildings Energy Consumption Survey (CBECS) database. International Journal of Energy Research, 2007. 31(4): p. 412-421.

12. Gao, $\mathrm{X}$. and $\mathrm{A}$. Malkawi, A new methodology for building energy performance benchmarking: An approach based on intelligent clustering algorithm. Energy and Buildings, 2014. 84: p. 607-616.

13. James Bergstra, R.B., Yoshua Bengio and Balázs Kégl, Algorithms for Hyper-Parameter Optimization. Energy Conversion and Management, 2011.

14. Jasper Snoek, H.L.a.R.P.A., Practical Bayesian Optimization of Machine Learning Algorithms. Energy Exploration \& Exploitation, 2012. 2019.

15. Huang, D., et al., Global Optimization of Stochastic Black-Box Systems via Sequential Kriging Meta-Models. Journal of Global Optimization, 2006. 34(3): p. 441-466.

16. DONALD R. JONES, M.S., and WILLIAM J.WELCH, Efficient Global Optimization of Expensive Black-Box Functions. Energy, 1998.

17. González, P.A. and J.M. Zamarreño, Prediction of hourly energy consumption in buildings based on a feedback artificial neural network. Energy and Buildings, 2005. 37(6): p. 595601.

18. Ruder, S., An overview of gradient descent optimization algorithms. arXiv, 2016.

19. Ba, D.P.K.a.J.L., ADAM: A METHOD FOR STOCHASTIC OPTIMIZATION. 3rd International Conference for Learning Representations, 2015.

20. Robinson, C., et al., Machine learning approaches for estimating commercial building energy consumption. Applied Energy, 2017. 208: p. 889-904. 